\title{
Endometriosis torácica como causa de neumotórax espontáneo recurrente en mujer de 53 años
}

\author{
Thoracic endometriosis presenting as recurrent spontaneous \\ pneumothorax in a 53-year-old female
}

\begin{abstract}
Emilio Pintor Holguin ${ }^{1}$, Margarita Rubio Alonso ${ }^{1}$, Mar Jiménez de la Peña² ${ }^{2}$ Benjamín Herreros Ruiz-Valdepeñas ${ }^{3}$. ${ }^{1}$ Universidad Europea de Madrid. ${ }^{2}$ Hospital Quirón Salud. Madrid. ${ }^{3}$ Hospital Universitario Fundación Alcorcón. Madrid.
\end{abstract}

\section{RESUMEN}

Una mujer de 53 años asintomática es diagnosticada de tercer episodio de neumotórax en hemitórax derecho. En episodios previos no se había objetivado causa del neumotórax salvo bullas subpleurales. Una videotoracoscopia muestra lesiones típicas de endometriosis torácica junto con múltiples perforaciones diafragmáticas. Tras la reparación quirúrgica la paciente recibió tratamiento anti-estrogénico con buena evolución. Se trata de un caso excepcional por su forma de presentación, por la edad de la paciente y por la afectación diafragmática.

Palabras clave: neumotórax espontáneo; neumotórax recurrente; neumotórax catamenial; endometriosis torácica.

\section{INTRODUCCIÓN}

El neumotórax espontáneo puede ser primario o secundario. Se denomina primario cuando ocurre en personas sin aparente patología pulmonar. Desde la introducción de la tomografía computarizada de alta resolución (HRCT) del tórax se ha podido comprobar que la mayor parte de los casos de neumotórax espontáneos primarios se producen por la rotura de una pequeña vesícula enfisematosa subpleural; Ilamada bleb o de una lesión enfisematosa subpleural paraseptal conocida como bulla'.

Entre las causas de neumotórax espontáneo secundario se encuentran patologías bronquiales como el asma, infecciosas como las neumonías necrotizantes, intersticiales pulmonares como la linfagioleiomatosis y la sarcoidosis, enfermedades del tejido conectivo como la artritis reumatoide, neoplasias malignas y endometriosis torácica².

En series largas y meta-análisis recientemente publicados ${ }^{3,4}$, en torno al 30-32\% de los pacientes tienen neumotórax espontáneos recurrentes. La mayor parte de las recurrencias ocurren en el periodo entre 6 meses y 2 después del primer episodio, siendo la recurrente más frecuente en mujeres.

Presentamos a continuación un caso de una mujer de 53 años que presentó 3 neumotórax espontáneos en los últimos 10 años. En los dos primeros; a pesar de toracoscopia y cirugía, solo se encontraron lesiones inespecíficas como blebs o bullas y se consideraron como primarios. En el tercer neumotórax; en la video-toracoscopia, se objetivaron lesiones típicas de endometriosis pleural y diafragmática con múltiples orificios diafragmáticos que producían herniaciones hepáticas llegando al diagnóstico de neumotórax espontáneo recurrente secundario a endometriosis pleural y diafragmática.

\section{ABSTRACT}

A 53-year-old asymptomatic female presented with a third episode of spontaneous pneumothorax in right hemithorax. Previous studies did not demonstrate the etiology of this disease but only sub-pleural bullae. We found typical thoracic endometriosis lesions through video thoracoscopy. After surgical correction and anti-estrogenic treatment, the patient remains asymptomatic. This case is relevant due its presentation, diaphragmatic involvement and the age of the patient.

Keywords: spontaneous pneumothorax; recurrent pneumothorax; catamenial pneumothorax; thoracic endometriosis.

\section{CASO CLÍNICO}

Paciente de 53 años, asintomática, a la que se realizó una radiografía de tórax de control por un neumotórax espontáneo previo (12 años antes) objetivándose un nuevo neumotórax encapsulado. Se realizó un TAC torácico que mostró un hidroneumotórax derecho encapsulado, así como herniación hepática transdiafragmática (Fig.1).

Entre los antecedentes personales de la paciente destacaban dos neumotórax espontáneos derechos a la edad de 39 y 40 años. En el primero, se practicó toracoscopia y grapado de bullas y en el segundo toracotomía, grapado de bullas y pleurodesis con povidona iodada. Estaba en periodo perimenopáusico y tenía 2 hijos. No había tenido nunca importante dismenorrea ni problemas de infertilidad.

Al ingreso hospitalario se encontraba eupneica con saturación basal de oxígeno del 97\% y con hipoventilación alveolar en base pulmonar derecha. Se procedió a inserción de drenaje torácico. Tras varios días sin resolución completa, se realizó cirugía mediante video-toracoscopia, observándose múltiples lesiones rojo-vinosas en pleura y diafragma, así como pequeñas perforaciones en el diafragma y una perforación de mayor tamaño por la que protruía el hígado. Se procedió a grapado de los orificios diafragmáticos y pleurodesis con talco. El estudio anatomo-patológico de las biopsias de diafragma y pleura revelaron la presencia de glándulas endometriales. La paciente fue diagnosticada de endometriosis torácica y de neumotórax catamenial recurrente. Se inició tratamiento trimestral con análogo de la hormona liberadora de gonadotropinas y a los 12 meses de seguimiento, la evolución radiológica y clínica ha sido favorable. 
Fig. 1. TAC tórax PA (A) y L (B). Se objetiva un neumotórax encapsulado en hemitoráx derecho (asteriscos) que diseca las cisuras y separa los 3 lóbulos (superior, medio e inferior). Pequeño derrame pleural y herniaciones hepáticas transdiafragmáticas (flechas).

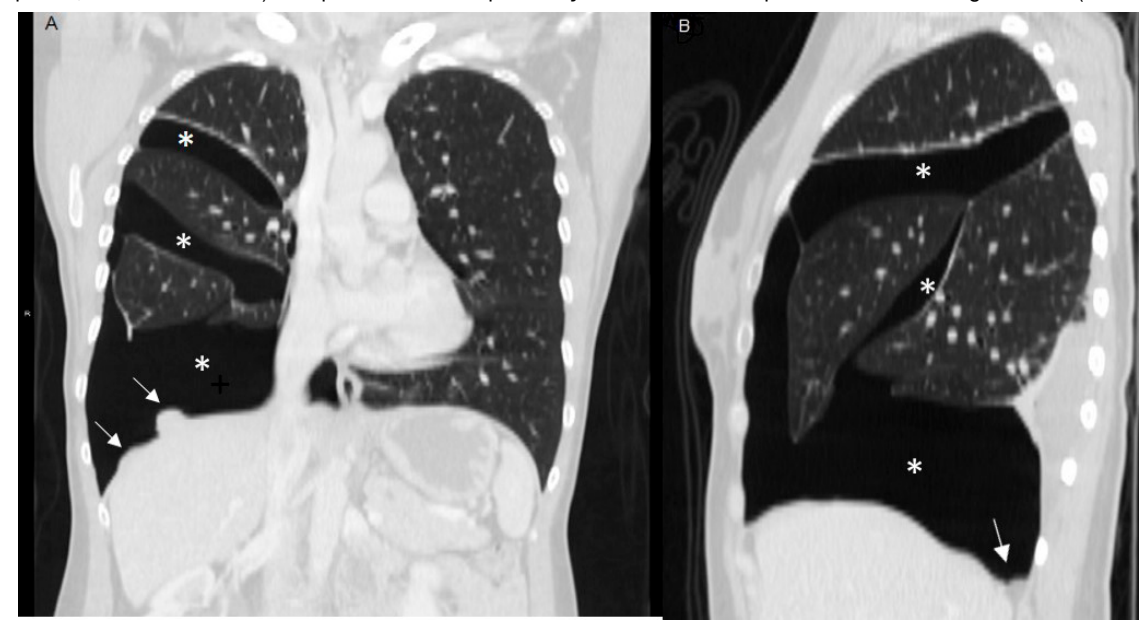

\section{DISCUSIÓN}

La endometriosis es una enfermedad caracterizada por la presencia de tejido endometrial ectópico (estroma y glándulas) fuera de la cavidad uterina. La cavidad abdominopélvica es el sitio más frecuente de afectación produciendo como sintomatología característica importante dismenorrea e infertilidad.

La cavidad torácica sería la zona de afectación más frecuente de endometriosis extra-pélvica. Puede localizarse en el parénquima pulmonar, en el diafragma 0 en la superficie pleural. Cuando en las lesiones histológicamente se identifican glándulas y estroma endometrial se considera endometriosis torácica probada y cuando solo se identifica solo estroma como probable 5 .

Clínicamente, la endometriosis torácica puede presentar un amplio rango de manifestaciones radiológicas y clínicas relacionadas con la menstruación: neumotórax catamenial (80\%), hemotórax catamenial (14\%), hemoptisis catamenial $(5 \%)$, y raramente nódulos pulmonares ${ }^{5}$.

El neumotórax catamenial es la forma más habitual de la endometriosis torácica. Ocurre mayoritariamente en mujeres entre los 30-40, aparece en los días en que la paciente tiene la menstruación, afecta con mayor frecuente el hemitórax derecho y suele recurrir con frecuencia, ${ }^{6,7,8,9}$.

Los hallazgos macroscópicos pueden incluir presencia de implantes de células endometriales en el diafragma y pleura, perforaciones diafragmáticas y rara vez herniación del hígado a través del diafragma ${ }^{9,10}$; como es el caso que presentamos.

Aunque varios tests diagnósticos pueden utilizarse (radiología de tórax, HRCT y resonancia nuclear magnética), en la actualidad la prueba más sensible es la toracoscopia videoasistida, encontrándose lesiones típicas en el diafragma (38.8\%) 0/y en la pleura visceral (29.6\%). Sin embargo, en casi la mitad de los casos en la visión quirúrgica se ven le- siones inespecificas como blebs o bullas (23.1\%), como se encontró en nuestra paciente en los 2 primeros neumotórax, y no se encontraron hallazgos en el $8.5 \%$ de los pacientes ${ }^{5}$. El tratamiento primario 0 agudo del neumotórax catamenial es el mismo que cualquier otro neumotórax espontáneo, el drenaje, pero debe ser complementado con tratamientos que prevengan la recurrencia del neumotórax, entre los que se encuentran la cirugía torácica con pleurodesis asociada así como cirugía pleuro-diafragmática cuando existen lesiones en el diafragma y el bloqueo hormonal mediante análogos de la GnRH (hormona liberadora de gonadotropina) ${ }^{5,8}$. Consideramos este caso excepcional tanto como por su forma de presentación clínica, como neumotórax recurrente, como por la edad de la paciente (53 años) así como por los hallazgos quirúrgicos inespecíficos en los 2 primeros neumotórax (blebs y bullas) y por la afectación diafragmática con herniación hepática.

\section{BIBLIOGRAFÍA}

1. Lyra RDM. Etiology of primary spontaneous pneumothorax. J Bras Pneumol.2016;42(3):222-6.

2. Shan A, Heffner J. Spontaneous pneumothorax. NEJM. 2000;342(12):868-74.

3. Walker SP, Bibby AC, Halford P, Stadon L, White P MN. Recurrence rates in primary spontaneous pneumothorax: a systematic review and meta-analysis. Eur Respir J. 2018;52((3)):1800864.

4. Hallifax R, Goldacre R, MJ L. Trends in the Incidence and Recurrence of InpatientTreated Spontaneous Pneumothorax, 1968-2016. JAMA. 2018;320(14):1471-80.

5. Nezhat C, Lindheim SR, Backhus L, Vu M. Thoracic Endometriosis Syndrome : A Review of Diagnosis and Management. JSLS. 2019;23(3):1-8.

6. Mehta CK, Stanifer BP, Fore-kosterski S, Gillespie C, Yeldandi A, Meyerson S, et al. Primary spontaneous pneumothorax in menstruating females has high recurrence. Ann Thorac Surg. 2016;102(4):1125-30.

7. Visouli AN, Zarogoulidis K, Kougioumtzi I, Huang H, Li Q, Dryllis G, et al. Catamenial pneumothorax. J Thorac Dis. 2014;6(24):448-60.

8. Junejo SZ, Lubana SS, Shina SS, Tuli SS. A Case of Thoracic Endometriosis Syndrome Presenting with Recurrent Catamenial Pneumothorax. Am J Case Rep. 2018;19:573-6

9. Rousset-jablonski C, Alifano M, Camilleri-broet S, Rousset P. Catamenial pneumothorax and endometriosis-related pneumothorax : clinical features and risk factors. Hum Reprod. 2011;26(9):2322-9.

10. Saito T, Saito Y, Fukumoto KJ et al. Clinical and Pathological Characteristics of Spontaneous Pneumothorax in Women: A 25-year Single-Institutional Experience. Gen Thorac Cardiovasc Surg. 2018;66((9)):516-522. 